\title{
Neue Erkenntnisse zu den Arbeitsmarkt- wirkungen internationaler Migration - Ein kritischer Überblick über vorliegende Befunde
}

Führt Migration zu steigender Arbeitslosigkeit und sinkenden Löhnen? Diese klassische Frage wird in diesem Beitrag vor dem Hintergrund neuer Erkenntnisse der Arbeitsmarkt- und Migrationsforschung behandelt. Denn nicht nur in der Bevölkerung, sondern auch unter Ökonomen ist die Annahme verbreitet, dass die Ausweitung des Arbeitsangebots durch Zuwanderung negative Folgen für die einheimischen Beschäftigten hat. Dies muss jedoch keineswegs so sein, allemal dann nicht, wenn sich Investitionen oder Gütermärkte anpassen. Empirisch lasst sich mittlerweile gut zeigen, ob und in welchem Umfang verschiedene Gruppen im Arbeitsmarkt durch Migration gewinnen oder verlieren. Neue methodische Ansätze haben hierzu in den letzten Jahren wichtige Erkenntnisse geliefert, die auch für Deutschland relevant sind.

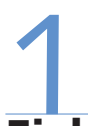

\section{Einleitung}

Die internationale Migration von Arbeitskräften ist eine häufig unterschätzte Dimension der Globalisierung. Angesichts anhaltend hoher Unterschiede in den Löhnen und Pro-Kopf-Einkommen zwischen den Ziel- und Herkunftsländern der Migration, fallender Transport- und Kommunikationskosten und institutioneller Veränderungen (wie beispielsweise die Freizügigkeit in der erweiterten Europäischen Union (EU) und die Lockerung der Zuwanderungsbedingungen für qualifizierte Arbeitskräfte) sind die Wanderungsraten in den vergangenen Dekaden weltweit gestiegen. In Europa nähert sich die jährliche Nettozuwanderungsrate mit rund drei Personen per Tausend an die der USA an (Weltbank 2009, vgl. dazu auch Abbildung 1). In Deutschland sind die Nettozuwanderungsraten nach ihrem sprunghaften Anstieg in den 1990er Jahren zwar seit der Jahrtausendwende stark gesunken. Dennoch ist Deutschland in absoluten Zahlen nach den USA und Russland das drittgrößte Einwanderungsland auf der Welt: Nach den Angaben des Mikrozensus haben $8 \%$ der Bevölkerung eine ausländische Staatsbürgerschaft, $12 \%$ sind im Ausland geboren und $19 \%$ haben einen Migrationshintergrund (Statistisches Bundesamt 2010)

\section{Abb. 1: Nettozuwanderung nach Deutschland, in die EU-15 und in die USA 1960-2008 - per 1.000 Personen -}

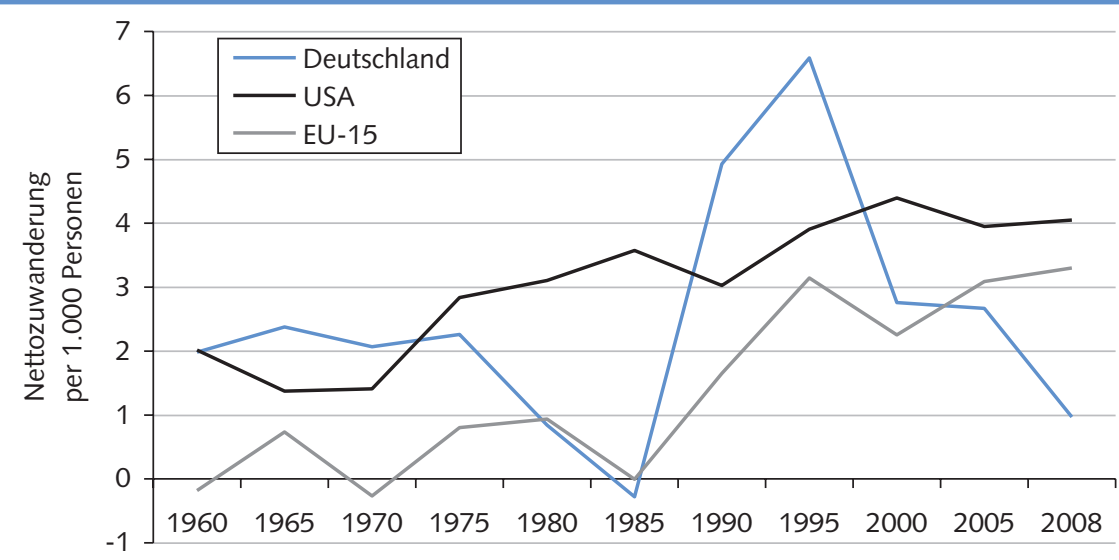

Quelle: Eurostat, UN Population Division, Statistisches Bundesamt, Berechnungen des Autors.

WSI MitTEILUNGEN

Die stärkere Zuwanderung nach Deutschland trifft auf erhebliche Ängste in der Bevölkerung und unter den Arbeitnehmern. Neben den Problemen der Integration von Zuwanderern stehen dabei die Folgen der Migration für die Arbeitsmärkte im Vordergrund. Führt die Ausweitung des Arbeitsangebots durch Migration zu fallenden Löhnen und, wenn die Löhne nicht vollkommen flexibel auf die Ausweitung des Arbeitsangebotes reagieren, zu steigender Arbeitslosigkeit? Diese Frage ist auch in der ökonomischen Literatur umstritten. In den vergangenen Jahren hat die empirische Forschung in den USA, aber auch in Europa, neue Ansätze entwickelt, um die Arbeitsmarktwirkungen der Migration zu messen. In diesem Beitrag werden die Auswirkungen der Migration

Herbert Brücker, Prof. Dr., lehrt an der Universität Bamberg und ist Leiter des Forschungsbereichs "Internationale Verg/eiche und Europäische Integration" beim Institut für Arbeitsmarkt- und Berufsforschung (IAB), Nürnberg. Arbeitsschwerpunkte: Internationale Migration, Arbeitsmärkte, Europäische Integration. Email: herbert.bruecker@iab.de 
auf Löhne und Beschäftigung untersucht. Aufbauend auf früheren Literatur-Surveys (Friedberg/Hunt 1995) werden hier die jüngsten Erkenntnisse der theoretischen, vor allem aber der empirischen Forschung berücksichtigt. ${ }^{1}$ Dabei werden zunächst vor dem Hintergrund unterschiedlicher Ansätze der Arbeitsmarkt- und Außenhandelstheorien verschiedene Hypothesen über die Arbeitsmarktwirkungen der Migration diskutiert, die als Ausgangspunkt für die empirische Forschung dienen (Abschnitt 2). Danach werden herkömmliche und neuere Ansätze in der empirischen Literatur vorgestellt, die die Lohn- und Beschäftigungswirkungen der Migration messen. Besonderes Augenmerk wird auf die Frage gelegt, ob diese Ansätze geeignet sind, die Migrationswirkungen vor dem Hintergrund von Arbeitsmärkten mit persistenter Arbeitslosigkeit abzuschätzen (Abschnitt 3). Schließlich werden wirtschaftspolitische Schlussfolgerungen gezogen (Abschnitt 4).

\section{Hypothesen der Arbeitsmarkt- und Außenhandelstheorien}

Auf den ersten Blick erscheint es einleuchtend, dass eine Ausweitung des Arbeitsangebotes zu sinkenden Löhnen oder, bei unvollkommenen Arbeitsmärkten, zu steigender Arbeitslosigkeit führt. Aus theoretischer Perspektive ist dieses Ergebnis jedoch nicht zwingend. Um die Wirkungsmechanismen der Migration zu untersuchen, wird hier zunächst der einfachste Fall diskutiert: die Zuwanderung in eine geschlossene Volkswirtschaft mit perfekten Arbeitsmärkten. Das entspricht dem neoklassischen Lehrbuchfall. Darauf aufbauend werden dann die Migrationswirkungen in Volkswirtschaften mit Lohnund Preisrigiditäten und struktureller Arbeitslosigkeit im Rahmen neokeynesianischer Arbeitsmarktmodelle untersucht.

\subsection{MIGRATION IN PERFEKTEN ARBEITSMÄRKTEN}

Betrachten wir zunächst die Folgen einer Ausweitung des Arbeitsmarktes in einer Volkswirtschaft mit räumenden Arbeitsmärkten, die keinen Handel betreibt und deren Ausstattung mit Kapital gegeben

\section{Abb. 2: Ausweitung des Arbeitsangebots durch Migration}
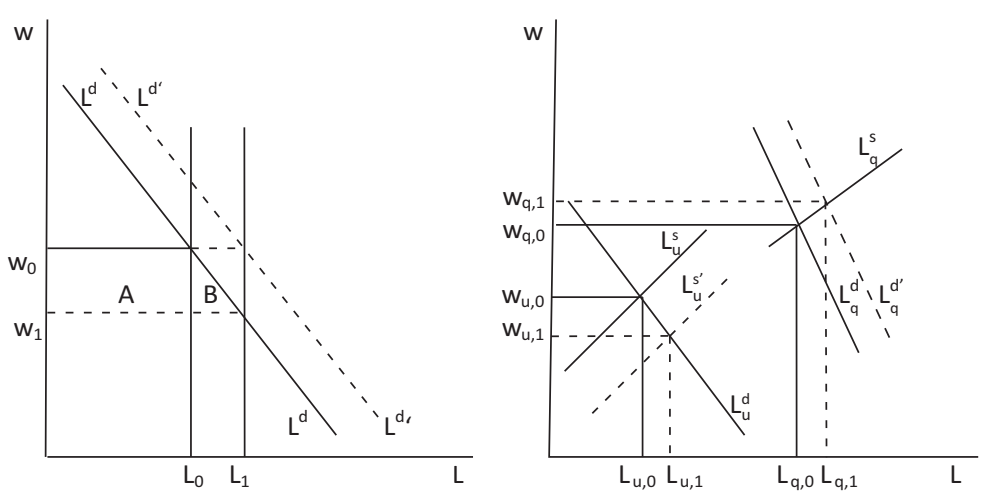

Quelle: Darstellung des Autors.

WSI MITTEILUNGEN

ist. Zur Vereinfachung nehmen wir auch an, dass das Arbeitsangebot unelastisch ist, d.h. nicht mit dem Lohn sinkt. Wie die linke Grafik in Abbildung 2 zeigt, führt die Ausweitung des Arbeitsangebots durch Migration von $L_{0} \mathrm{zu} L_{1}$ dazu, dass der Lohn von $w_{0}$ auf $w_{1}$ fällt. Die bereits im Lande lebenden Arbeitnehmer verlieren die rechteckige Fläche A. Die Unternehmen profitieren von sinkenden Löhnen und der Ausweitung der Produktion, sodass sich bei einer fixen Kapitalausstattung und der fallenden Arbeitsnachfragekurve $L^{d}$ ein Anstieg der Unternehmenseinkommen um die Flächen A + B ergibt. Insgesamt ergibt sich für die einheimische Bevölkerung - unter der Annahme, dass ihr der Kapitalstock gehört - ein Nettoeinkommensgewinn in Höhe der Fläche B. Für die Herkunftsländer gilt der umgekehrte Fall. Dort würden die Löhne steigen, die Kapitalrendite fallen und das Nationaleinkommen der zurückbleibenden Bevölkerung sinken. Sie könnten nur profitieren, wenn die Migranten einen Teil ihres im Zielland erwirtschafteten Einkommens ins Herkunftsland zurücküberweisen (vgl. z. B. Wong 1995).

Nun ist die Annahme unrealistisch, dass Investoren nicht auf die Ausweitung des Arbeitsangebotes reagieren würden und dass der Kapitalstock konstant bleibt. Da die Kapitalrendite steigt, investieren profitmaximierende Unternehmen und passen den Kapitalstock an. Das gilt für den Standardfall eines neoklassischen Wachstumsmodells mit konstanter Sparrate. Bei internationaler Kapitalmobilität wäre zu erwarten, dass diese Anpassung noch schneller erfolgt. In unserer Grafik würde sich in diesem Fall die Arbeitsnach- fragekurve von $L^{d}$ nach $L^{d^{\prime}}$ verschieben. Bei einer vollständigen Anpassung des Kapitalstocks bliebe der Lohn gleich bei $\mathrm{w}^{0}-\mathrm{es}$ ergäbe sich kein Einkommensverlust für die Arbeitnehmer.

Diese Überlegungen mögen auf den ersten Blick theoretisch klingen. Tatsächlich gehört es jedoch zu den wenigen belastbaren Fakten in der empirischen Wirtschaftsforschung, dass das Verhältnis von Kapital zu Output und damit die Kapitalintensität der Produktion unter Berücksichtigung des Produktivitätsfortschritts langfristig konstant bleiben. Dies wurde von Nicholas Kaldor bereits vor fast 50 Jahren beschrieben (Kaldor 1961) und seitdem durch zahlreiche Studien immer wieder bestätigt. Abbildung 3 zeigt diesen Zusammenhang für Westdeutschland: Obwohl die Zahl der Erwerbspersonen von 26 Mio. Personen im Jahr 1960 auf 35,5 Mio. Personen im Jahr 2005 gestiegen ist, ist das Verhältnis von Kapital zu Output bei einem Faktor von rund drei konstant geblieben. Das hat erhebliche Implikationen für die Migration: Zumindest langfristig würden sich durch die Anpassung des Kapitalstocks keine Folgen der Zuwanderung für das gesamtwirtschaftliche Lohnniveau ergeben. Es ist auch zu berücksichtigen, dass Migration häufig nicht als unerwarteter Schock, sondern kontinuierlich auftritt, sodass die Investoren ihre Erwartungen darauf einstellen können. Dies würde erklären, dass die Ka-

1 Zu einer jüngeren Darstellung vgl. auch Brücker (2009). Im Vergleich zu diesem populärwissenschaftlichen Beitrag werden im vorliegenden Text die Probleme der verschiedenen Ansätze und ihre Ergebnisse sehr viel detaillierter behandelt. 


\section{Abb. 3: Kapital-Output-Ratio und Erwerbspersonen in Westdeutschland 1960-2008}

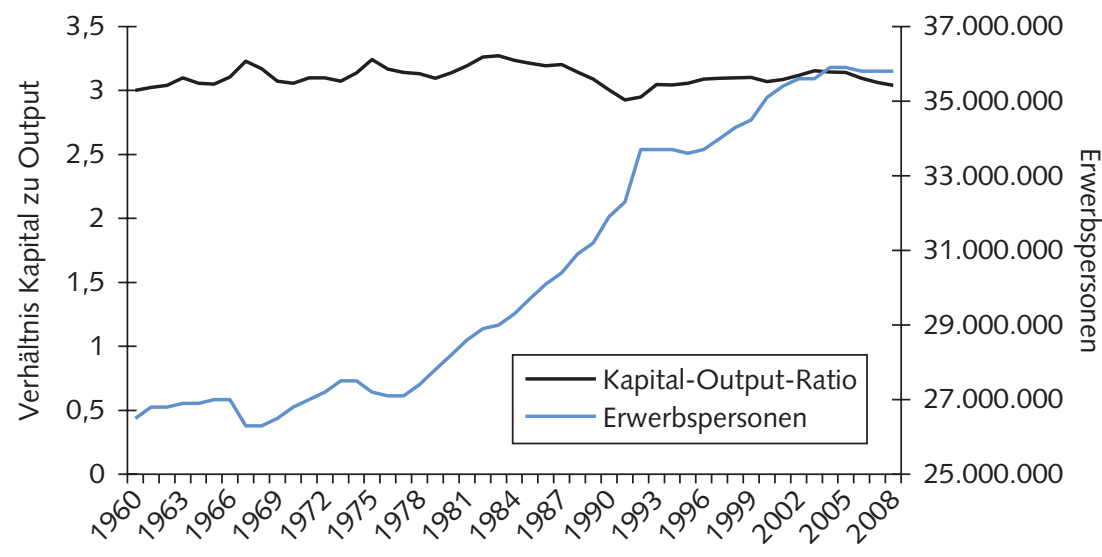

Anmerkung: Der Kapitalstock wird durch das Bruttoanlagevermögen gemessen, der Output durch das Bruttoinlandsprodukt.

Quelle: Berechnungen des Autors auf Grundlage von Daten der OECD (2010).

WSI MITTELLUNGEN

pitalintensität der Produktion auch kurzfristig nicht wesentlich durch Migration beeinflusst wird.

In einer offenen Volkswirtschaft passen sich nicht nur die Kapitalmärkte, sondern auch die Gütermärkte an Veränderungen des Arbeitsangebots an. Im Standardmodell der Außenhandelstheorie, dem Heckscher-Ohlin-Samuelson-Modell, wird jede Volkswirtschaft diejenigen Güter exportieren, für deren Produktion diejenigen Faktoren intensiv eingesetzt werden, mit denen sie relativ reichlich ausgestattet ist. Und sie wird diejenigen Güter importieren, für deren Produktion Faktoren eingesetzt werden, mit denen sie relativ knapp ausgestattet ist. Die Ausweitung des Arbeitsangebots durch Zuwanderung würde dann dazu führen, dass der Anteil der arbeitsintensiven Produktion ausgeweitet und jener der kapitalintensiven Produktion verringert würde (Rybczynski-Effekt). Die Volkswirtschaft würde weniger arbeitsintensive Güter importieren und weniger kapitalintensive Güter exportieren. Im Falle eines kleinen Landes, für das die Preise auf den Weltmärkten gegeben sind, bleiben die Preise auf den Gütermärkten unverändert. Dies wiederum bewirkt, dass auch die Faktorpreise, d.h. der Lohn und die Kapitalrendite, trotz der Ausweitung des Arbeitsangebotes konstant bleiben.

Bislang sind wir davon ausgegangen, dass Arbeit ein homogener Faktor ist. Tatsächlich unterscheidet sich Arbeit in vielen Dimensionen - durch Ausbildung und Beruf, Berufserfahrung, Alter, Sprachkompetenz und andere Faktoren wie kulturelle Eigenschaften und ethnischer Hintergrund. Die rechte Grafik in Abbildung 2 illustriert den Fall der Zuwanderung, wenn zwei Gruppen im Arbeitsmarkt existieren, gering qualifizierte und qualifizierte Arbeitskräfte. Wir berücksichtigen zudem den Fall eines elastischen Arbeitsangebots, d.h. dass mit fallendem Lohn das Arbeitsangebot zurückgeht und die Arbeitslosigkeit steigt. Durch Migration steigt in diesem Beispiel das Angebot gering qualifizierter Arbeit. Die Arbeitsangebotskurven für gering qualifizierte $\mathrm{Ar}$ beit verschieben sich von $L_{u}{ }^{s}$ nach $L_{u}{ }^{{ }^{\prime}}$. Der Lohn für gering qualifizierte Arbeit fällt von $w_{u, 0}$ auf $w_{u, 1}$, die beschäftigte Arbeit steigt von $L_{u, 0}$ auf $L_{u, 1}$, aber um weniger als die Zahl der zugewanderten Arbeitskräfte. Das ist darauf zurückzuführen, dass auch bei fallendem Lohn das Arbeitsangebot fällt. Mit der Ausweitung des Arbeitsangebotes steigt die Produktion. Für die anderen Arbeitsmarktsegmente - in unserem Fall qualifizierte Arbeit - ergeben sich zwei Effekte: Erstens, steigt mit der Ausweitung der Produktion die Arbeitsnachfrage. Zweitens, kann qualifizierte Arbeit durch die günstiger gewordene unqualifizierte Arbeit substituiert werden, sodass sich bei gegebener Produktion die Arbeitsnachfrage reduzieren würde (vgl. Hamermesh 1993).

Welcher der beiden Effekte überwiegt, ist eine empirische Frage. Ingenieure etwa können nur in geringem Umfang durch ungelernte manuelle Arbeitskräfte ersetzt werden, sodass sie mit hoher Wahrscheinlichkeit von der Zuwanderung gering qualifizierter Arbeitskräfte profitieren werden. Umgekehrt können ungelernte Arbeitskräfte möglicherweise stärker Facharbeitertätigkeiten substituieren. In der rechten Grafik von Abbildung 2 wird davon ausgegangen, dass die Ausweitung des Arbeitsangebots ungelernter Arbeitskräfte die Nachfrage nach qualifizierter Arbeit ausweitet, mit der Folge, dass der Lohn für qualifizierte Arbeit steigt und bei gegebener Arbeitsangebotskurve die Beschäftigung von qualifizierter Arbeit zunimmt.

\subsection{MIGRATION IN VOLKSWIRT- SCHAFTEN MIT LOHNRIGIDITÄTEN UND ARBEITSLOSIGKEIT}

Die bisher diskutierten Modelle gehen von der Annahme perfekter Arbeitsmärkte aus, d.h. von flexiblen Löhnen und räumenden Märkten. In diesem Fall kann zwar das Arbeitsangebot durch fallende Löhne sinken, Arbeitslosigkeit ist aber freiwillig. Die wichtigste Befürchtung im Kontext von Migration bezieht sich jedoch auf die Frage, ob die Ausweitung des Arbeitsangebots durch Zuwanderung zu einem Anstieg der Arbeitslosigkeit führt. Solche Bedenken waren Anlass für den Migranten-Anwerbestopp nach dem ersten Ölpreisschock 1973 und sie motivieren die seitdem verfolgte restriktive Zuwanderungspolitik in Deutschland. Aus neoklassischen Modellen mit räumenden Arbeitsmärkten können deshalb schwer wirtschaftspolitisch relevante Aussagen abgeleitet werden hierfür sind Modelle notwendig, die Arbeitslosigkeit berücksichtigen.

Die Makroökonomie und Arbeitsmarkttheorie bieten zahlreiche Erklärungen für die Phänomene von friktioneller, struktureller und zyklischer Arbeitslosigkeit an, die alle einen Beitrag zum Verständnis der Auswirkungen der Migration auf Arbeitslosigkeit leisten können. Hier werden die Auswirkungen der Migration im Rahmen neukeynesianischer Modelle diskutiert, die Preis- und Lohnrigiditäten berücksichtigen (Layard et al. 2005). Diese Modelle ersetzen die herkömmliche Arbeitsangebotsfunktion durch eine Lohnsetzungskurve („wage-setting curve"), die unterstellt, dass der Lohn bei steigender Arbeitslosigkeit fällt. Dieser makroökonomische Zusammenhang kann empirisch beobachtet werden. Allerdings reagieren die Löhne nicht vollkommen elastisch und mit Zeitverzögerungen auf einen Anstieg 
der Arbeitslosigkeit, sodass in diesen Modellen sowohl zyklische wie auch strukturelle Arbeitslosigkeit erklärt werden kann. Mikroökonomisch kann die Existenz einer solchen Lohnsetzungskurve durch sehr unterschiedliche theoretische Ansätze fundiert werden, etwa durch Effizienzlohntheorien, kollektive Lohnverhandlungen zwischen Gewerkschaften und Arbeitgebern sowie Search- und Matchingtheorien. ${ }^{2}$

Inzwischen hat eine Reihe von Beiträgen Lohnsetzungsmodelle für die Analyse der Arbeitsmarktwirkungen der Migration verwendet (Boeri/Brücker 2005; Brücker/ Jahn 2010; Levine 1999). Die Wirkungen der Migration auf die Arbeitslosigkeit sind zwiespältig. Im Falle von nur einem Typ von Arbeit würde die Ausweitung des Arbeitsangebots sowohl einen Rückgang der Löhne als auch einen Anstieg der Arbeitslosigkeit bewirken, sofern sich die Löhne nicht vollkommen flexibel an die Ausweitung des Arbeitsangebots anpassen. Im Falle unterschiedlicher Typen von Arbeit kann die Zuwanderung jedoch auch die Arbeitslosigkeit senken: Die Effekte hängen davon ab, wie die Löhne in den Arbeitsmarktsegmenten reagieren, in denen das Arbeitsangebot ausgeweitet wird. Sind die Löhne in den Segmenten flexibler, in denen das Arbeitsangebot durch Migration überdurchschnittlich ausgeweitet wird, kann die Arbeitslosigkeit auch sinken. Natürlich ist auch der umgekehrte Fall denkbar (vgl. Boeri/Brücker 2005; Brücker/Jahn 2010).

Komplizierter liegt der Fall, wenn die Migration selbst den Lohnsetzungsmechanismus beeinflusst. Dies kann etwa dadurch geschehen, dass sich das Arbeitsangebotsverhalten der Migranten aufgrund der hohen Einkommensdifferenzen zu den Herkunftsländern von denen der einheimischen Bevölkerung unterscheidet, sodass ihr Lohn flexibler auf einen Anstieg der Arbeitslosigkeit reagiert. Es ist ebenfalls denkbar, dass sie durch einen geringeren Organisationsgrad oder Lohndumping die Verhandlungsmacht der Gewerkschaften schwächen. Dann würde Migration zu fallenden Löhnen, einer höheren Lohnflexibilität, aber auch zu einer geringeren Arbeitslosigkeit führen. Zugleich würde die Steigung der Phillips-Kurve, die den Zusammenhang zwischen Inflation und Arbeitslosigkeit abbildet, flacher verlaufen, womit die Handlungsspielräume für eine expansive makroökonomische Politik steigen (Binyamini/Razin 2008; Bentolila et al. 2007). Ob dies tatsächlich der Fall ist, ist noch nicht hinreichend geklärt die Europäischen Zentralbanken verfolgen diese Frage jedoch mit großem Interesse. Eine spanische Studie scheint zu belegen, dass die Verschiebung der Lohnsetzungsund Phillips-Kurve erklärt, warum es bei einer Zuwanderung von knapp $10 \%$ der Bevölkerung in Spanien in wenigen Jahren dort gleichzeitig zu einem Rückgang der Arbeitslosigkeit um sechs Prozentpunkte gekommen ist (Bentolila et al. 2007). Es wäre folglich denkbar, dass Migration auch einen Rückgang der Arbeitslosigkeit bewirkt - auch wenn dies zumindest in Teilen des Arbeitsmarktes mit Lohnsenkungen erkauft wird.

\section{Neue und alte empirische
Erkenntnisse}

Die empirische Literatur zu den Arbeitsmarktwirkungen der Migration hat seit Mitte der vergangenen Dekade durch methodische Innovationen einen erheblichen Aufschwung genommen. Doch auch bereits in den 1980er und 90er Jahren gab es einen umfassenden Literaturstand von mehreren hundert Studien, die in den USA und Europa durchgeführt worden sind und versucht haben, die Arbeitsmarktwirkungen der Migration zu messen. Die jüngeren Studien bauen hierauf auf.

\subsection{TRADITIONELLE SCHÄTZANSÄTZE}

Der überwiegende Teil der empirischen Literatur nutzt die Varianz des Ausländeranteils über die verschiedenen Regionen eines Landes, um die Arbeitsmarktwirkungen der Migration zu identifizieren. In diesen Regressionsanalysen werden die Löhne oder Beschäftigungsraten (bzw. Arbeitslosenraten) durch den Ausländeranteil und verschiedene Kontrollvariablen, die den Einfluss anderer Faktoren ausschließen sollen, erklärt. Unter der starken Annahme, dass der Ausländeranteil nicht mit der zu erklärenden Variable, also dem Lohn oder der Beschäftigungsrate in einer Region, korreliert ist, lassen sich so die Arbeitsmarktwirkungen der Migration auf diesem Weg korrekt identifizieren (Borjas et al. 1997). Genau diese Annahme ist jedoch in der Regel nicht erfüllt: Migranten wandern in diejenigen Regionen, in denen die Löhne besonders hoch und die Arbeits- losenraten besonders niedrig sind. So sind die Migranten in Deutschland insbesondere in den süddeutschen Ballungsräumen, dem Rhein-Main-Gebiet und in Hamburg konzentriert. Dort sind die Löhne überdurchschnittlich hoch und die Arbeitslosenraten liegen unter dem Bundesdurchschnitt, während beispielsweise auf Berlin mit seiner hohen Arbeitslosigkeit nur ein unterdurchschnittlicher Migrantenanteil unter den deutschen Großstädten entfällt. Wenn die Migranten jedoch nicht zufällig über den Raum verteilt sind, dann können sich verzerrte und inkonsistente Schätzergebnisse ergeben. Eine naive Schätzgleichung, die einfach den Lohn oder die Arbeitslosenrate gegen den Ausländeranteil regressiert, könnte deshalb zu dem Ergebnis kommen, dass durch Migration die Löhne steigen und die Arbeitslosenrate sinkt.

In den empirischen Studien, die die regionale Varianz des Ausländeranteils nutzen, werden deshalb zwei unterschiedliche Strategien verfolgt, um verzerrte Schätzungen zu vermeiden: Der erste Teil stützt sich auf natürliche Experimente, in denen die Migration durch politische Ereignisse und nicht durch ökonomische Motive gesteuert wurde. Das bekannteste Beispiel ist der Mariel Boatlift von 1982, eine Massenemigration von Kubanern in die Region Miami, die durch eine überraschende Ausreiseerlaubnis Fidel Castros ermöglicht wurde. Rund die Hälfte dieser Migranten hat sich in Miami niedergelassen, wodurch dort die Bevölkerung um rund $8 \%$ stieg. Der amerikanische Arbeitsökonom David Card (1990) hat dieses natürliche Experiment untersucht und keinen signifikanten negativen Zusammenhang zwischen der Zuwanderung von Kubanern und den Löhnen und Beschäftigungschancen vergleichbarer Gruppen in der Region Miami gefunden. Vergleichbare Studien haben andere natürliche Experimente, wie die Rückkehr von Franzosen nach Frankreich nach dem Ende des Algerienkriegs (Hunt 1992) und die Rückkehrmigration aus den portugiesischen Kolonien Mozambique und Angola nach der Nelkenrevolution von 1974 (Carrington/DeLima 1996), genutzt. Die meisten dieser Studien finden keine oder nur geringe Lohn- und Beschäftigungseffekte

\footnotetext{
2 Diese Modelle können als "neukeynesianisch" bezeichnet werden, weil sie eine mikroökonomische Fundierung für makroökonomische Preisund Lohnrigiditäten anbieten.
} 


\begin{tabular}{|c|c|c|c|}
\hline \multicolumn{4}{|c|}{ Übersicht 1: Lohn- und Beschäftigungseffekte der Zuwanderung: Ergebnisse regionaler Regressionsanalysen } \\
\hline \multicolumn{4}{|c|}{ Metastudien } \\
\hline Longhi et al. (2008) & 854 Effekte in 21 Ländern & $-0,10$ & - \\
\hline Longhi et al. (2006) & 165 Effekte in 7 Ländern & - & $-0,024$ \\
\hline \multicolumn{4}{|c|}{ Ausgewählte internationale Studien } \\
\hline Card (1990) & $\begin{array}{l}\text { Miami im Vergleich zu anderen US-Regionen } \\
\text { (natürliches Experiment) }\end{array}$ & keine Korrelation & keine Korrelation \\
\hline Borjas et al. (1997) & Regionen und Staaten, USA & $\begin{array}{l}-0,11 \text { bis } \\
-0,21\end{array}$ & - \\
\hline Hunt (1992) & Regionen in Frankreich (natürliches Experiment) & $\begin{array}{l}0,00 \text { bis } \\
-0,80\end{array}$ & bis $z u-0,20$ \\
\hline Carrington/DeLima (1996) & Regionen in Portugal (natürliches Experiment) & $\begin{array}{l}-0,15 \text { bis } \\
-2,50\end{array}$ & - \\
\hline Winter-Ebmer/Zweimüller (1996) & Regionen in Österreich & insignifikant & insignifikant \\
\hline Dustmann et al. (2005) & Regionen in GB & insignifikant & insignifikant \\
\hline \multicolumn{4}{|c|}{ Ausgewählte deutsche Studien } \\
\hline DeNew/Zimmermann (1994) & Regionen in Deutschland & $\begin{array}{l}0,00 \text { bis } \\
-0,10\end{array}$ & - \\
\hline $\begin{array}{l}\text { Haisken-DeNew/ } \\
\text { Zimmermann (1995) }\end{array}$ & Regionen in Deutschland & 0,00 & - \\
\hline 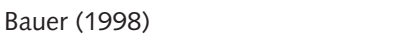 & Arbeitsmarktregionen in Deutschland & 0,00 & - \\
\hline Glitz (2006) & Spätaussiedler, Regionen in Deutschland & $-0,06$ & $-0,13$ \\
\hline Pischke/Velling (1997) & 167 Raumordnungsregionen in Deutschland & - & $\begin{array}{l}\text { insignifikant, } A L-\text { Rate } \\
\text { zwischen } 0,00 \text { und } 0,20\end{array}$ \\
\hline Winter-Ebmer/Zimmermann (1998) & Industriebranchen in Deutschland & $-0,02$ & 0,025 \\
\hline Mühleisen/Zimmermann (1994) & Regionen in Deutschland & - & insignifikant \\
\hline Winkelmann/Zimmermann (1993) & Regionen in Deutschland & - & insignifikant \\
\hline \multicolumn{4}{|c|}{$\begin{array}{l}\text { 1) Veränderung in \% bei einer Erhöhung des Ausländeranteils um einen Prozentpunkt. 2) Veränderung in Prozentpunkten bei einer Erhöhung des } \\
\text { Ausländeranteils um einen Prozentpunkt. Die Effekte wurden durch eigene Umrechnungen normiert. }\end{array}$} \\
\hline \multicolumn{3}{|c|}{ Quelle: Eigene Zusammenstellungen und Berechnungen auf Grundlage der o.g. Studien. } & MITTEILUNGEN \\
\hline
\end{tabular}

der Zuwanderung. In Deutschland wurde in einer jüngeren Studie die administrative Verteilung von Spätaussiedlern aus der früheren Sowjetunion über die Regionen der Bundesrepublik als natürliches Experiment ausgewertet (Glitz 2006). Diese Studie findet ebenfalls keine signifikanten Lohneffekte, aber nicht unerhebliche Beschäftigungseffekte.

Natürliche Experimente sind vergleichsweise selten. Der überwiegende Teil der Literatur verwendet deswegen Schätzverfahren mit Instrumenten, um unverzerrte und konsistente Ergebnisse zu erreichen. Instrumente sind Variablen, die mit der erklärenden Variable, nicht aber den zu erklärenden Variablen korreliert sind. Bei einer korrekten Verwendung von Instrumenten kann in ökonometrischen Studien die statistische Verzerrung aufgrund eines Endogenitätsproblems korrigiert werden. Solche Instrumente zu finden, ist schwer. In unserem Fall müssen Variablen gefunden werden, die mit dem Ausländeranteil, nicht aber den Löhnen oder Beschäftigungsraten in einer Region korreliert sind. Üblicherweise wird der verzögerte Ausländeranteil als Instrument für den aktuellen Ausländeranteil in der Literatur verwendet. Es ist jedoch kontrovers, ob dies tatsächlich ein geeignetes
Instrument ist, weil der verzögerte Migrationsanteil in einer Region ähnlich mit der Lohn- und Beschäftigungsentwicklung dieser Region korreliert sein kann wie der aktuelle Migrationsanteil. Dies gilt umso mehr, wenn die Akteure Erwartungen über die Lohn- und Beschäftigungsentwicklung in einer Region entwickeln. ${ }^{3}$

Untersuchungen, die sich auf natürliche Experimente stützen können, gelten deshalb methodisch als belastbarer. Aber auch dies wird in den letzten Jahren zunehmend kritisiert. Alle Schätzstrategien, die die regionale Varianz des Ausländeranteils zwischen den Regionen zur Identifikation der Arbeitsmarktwirkungen nutzen, können die Effekte nur dann korrekt identifizieren, wenn sie auf die betroffene Region beschränkt bleiben. Das ist jedoch fraglich. So kann die Zuwanderung von Migranten zu einer Abwanderung von Einheimischen oder zu einer geringeren Zuwanderung von anderen betroffenen Gruppen in die betroffene Region führen (Borjas et al. 1997; Borjas 2003). Allerdings lassen sich für dieses Argument wenig stichhaltige empirische Belege vorbringen - eine signifikante Veränderung des Wanderungsverhaltens nach einem Migrationsschock kann statistisch schwer nachgewiesen werden (Card/ Di Nardo 2000; Card 2001; Card 2005).
Gewichtiger ist das Argument, dass die Wirkungen der Zuwanderung in einer Region durch Handel und Kapitalmobilität auch auf andere Regionen verteilt werden. Eine Regression, die die regionale Varianz des Ausländeranteils nutzt, würde die Migrationswirkungen dann systematisch unterschätzen (Borjas 2003). Dies wäre aus Sicht der Handelstheorie tatsächlich zu erwarten.

Trotz dieser methodischen und empirischen Einwände haben mehrere hundert Studien in den USA, Deutschland und anderen europäischen Ländern die Varianz des Ausländeranteils genutzt, um die Arbeitsmarktwirkungen der Migration zu identifizieren. Übersicht 1 fasst die Ergebnisse einiger ausgewählter internationalen und deutschen Studien zusammen. Wie wir sehen, findet der überwiegende Teil der Literatur keine oder nur sehr geringe Lohn- und Beschäftigungseffekte. Wie die Meta-Studien von Longhi et al. (2005, 2006, 2008) zeigen, reduziert der Anstieg des Ausländeranteils um $1 \%$ der

3 Eine detaillierte Diskussion der ökonometrischen Probleme, die sich aus der Endogenität der Zuwanderung ergeben, übersteigt den Rahmen dieses Beitrags. Vgl. z.B. Wooldrige (2007) für eine vertiefende Diskussion. 
Bevölkerung (das entspricht in Deutschland 820.000 Personen) die Löhne um rund $0,1 \%$ und reduziert die Beschäftigungsrate der einheimischen Bevölkerung bzw. der gesamten Bevölkerung nur um 0,026 Prozentpunkte. Diese geringen Effekte werden nicht nur in den US-amerikanischen Studien, sondern auch in vielen deutschen Studien ermittelt: So schwankt die Lohnelastizität in den meisten Studien zwischen 0,00 und $-0,10$ und die Elastizität der Beschäftigung in den meisten Studien um null. Die wohl methodisch anspruchvollste Studie zur Messung der Beschäftigungswirkungen von Pischke/Velling (1997) findet keine signifikanten Effekte der Migration auf die Beschäftigungsrate, während die Effekte für die Arbeitslosenrate je nach Spezifikation des Schätzmodells zwischen null und maximal 0,2 schwanken.

Insgesamt kann aus der traditionellen Literatur, die die regionale Varianz des Ausländeranteils nutzt, also die Schlussfolgerung gezogen werden, dass die Arbeitsmarktwirkungen der Migration entweder neutral oder gering sind. Aufgrund der methodischen Probleme der Identifikation der Migrationseffekte sind diese Studien jedoch seit Ende der 1990er Jahre einer wachsenden Kritik ausgesetzt.

\subsection{NEUE SCHÄTZSTRATEGIEN}

Vor dem Hintergrund der methodischen Probleme in der bestehenden Literatur hat Borjas (2003) vorgeschlagen, anstelle der Varianz des Ausländeranteils zwischen den Regionen die Varianz des Ausländeranteils über einzelne Qualifikations- und Berufserfahrungsgruppen auf nationaler Ebene für die Identifikation der Arbeitsmarktwirkungen der Migration zu nutzen. Unter der Annahme, dass die Zuwanderung nicht durch Lohnunterschiede in den einzelnen Qualifikations- und Berufserfahrungsgruppen des Arbeitsmarktes beeinflusst wird, hätte diese Schätzstrategie den Vorteil, dass sich kein Endogenitätsproblem ergibt. Zwar mögen Unterschiede in der Entlohnung zwischen den einzelnen Berufsgruppen eine geringfügige Rolle bei Migrationsentscheidungen spielen, insgesamt ist diese Annahme jedoch durchaus plausibel. In seiner Studie kommt Borjas (2003) zu sehr viel höheren Schätzergebnissen für die USA als die frühere Literatur: Die Zuwanderung von einem Prozent der Erwerbspersonen führt bei ihm zu einem
Rückgang der durchschnittlichen Löhne um 0,3 bis $0,4 \%$. Besonders betroffen sind gering qualifizierte Arbeitskräfte. Diese Ergebnisse wurden von ihm in einer weiteren Studie für Kanada und Mexiko bestätigt (Aydemir/Borjas 2005).

Die Ergebnisse von Borjas und seine Schätzstrategie sind wenige Jahre später jedoch durch eine Arbeit von Ottaviano/ Peri (2006) herausgefordert worden. Ihre Studie stützt sich auch auf einen nationalen Schätzansatz, unterscheidet sich aber durch zwei Merkmale von der Arbeit von Borjas (2003): Erstens wird die Anpassung des Kapitalstocks berücksichtigt, in dem die Anpassungsgeschwindigkeit des Kapitalstocks geschätzt wird. Ihre Schätzergebnisse bestätigen die Hypothese von Kaldor (1961), dass das Verhältnis von Kapital zu Output langfristig konstant ist. Zudem passt sich der Kapitalstock in wenigen Jahren an Arbeitsangebotsschocks an. Zweitens berücksichtigt die Studie, dass sich Arbeit nicht nur in Hinblick auf die Ausbildung und Berufserfahrung, sondern auch in Hinblick auf nationale oder ethnische Herkunft unterscheiden kann. Dafür sprechen Argumente wie unterschiedliche Sprachkompetenz, ethnische und kulturelle Unterschiede usw. Ihre Schätzergebnisse zeigen, dass Ausländer und Inländer imperfekte Substitute im Arbeitsmarkt sind, d.h. auch bei gleicher Ausbildung und Berufserfahrung nur unvollkommen im Arbeitsmarkt konkurrieren. Die Berücksichtigung dieser beiden Aspekte hat weitreichende Folgen: Erstens sind die aggregierten Arbeitsmarkteffekte der Migration aufgrund der Anpassung des Kapitalstocks sehr viel geringer als bei Borjas und vergleichbar mit den Größenordnungen der herkömmlichen Literatur. Zweitens gewinnen die meisten einheimischen Arbeitskräfte durch Zuwanderung, während die bereits im Lande lebenden Ausländer erheblich verlieren (Ottaviano/ Peri 2006).

Ob Ausländer und Inländer tatsächlich perfekte oder imperfekte Substitute im Arbeitsmarkt sind, ist umstritten. Während Borjas et al. (2008) behaupten, dass die Ergebnisse von Ottaviano/Peri (2006) auf einer Fehlklassifizierung der Qualifikationsund Berufserfahrungsgruppen beruhen, zeigen Ottaviano/Peri (2008), dass auch bei anderen Klassifizierungen Aus- und Inländer tatsächlich imperfekte Substitute im Arbeitsmarkt sind. Die neuen Ansätze der US-Literatur werden auch von einer
Reihe neuer europäischer Studien, vor allem in Deutschland und Großbritannien, angewandt. Diese Studien bestätigen fast alle das Ergebnis von Ottaviano/Peri (2006, 2008), dass Aus- und Inländer unvollkommene Substitute im Arbeitsmarkt sind (vgl. Brücker/Jahn 2010, D’Amuri et al. 2008, Felbermayr et al. 2008, Manacorda et al. 2006).

Übersicht 2 fasst die Ergebnisse der neueren Studien zusammen. Zu unterscheiden sind die kurzfristigen Effekte, also die Effekte der Migration, wenn sich der Kapitalstock noch nicht an die Ausweitung des Arbeitsangebots angepasst hat, von den langfristigen Effekten, die sich nach der Anpassung des Kapitalstocks ergeben. Die langfristigen Effekte werden in Klammern angegeben. Während Borjas (2003) und Aydemir/Borjas (2005) schätzen, dass ein Anstieg des Ausländeranteils um einen Prozentpunkt kurzfristig die Löhne um 0,3 bis $0,4 \%$ senkt, kommen Ottaviano/ Peri $(2006,2008)$ in ihren Studien für die USA zu dem Ergebnis, dass der Lohn nur um $0,1 \%$ fällt. Auch die deutschen Studien ermitteln, dass ein Anstieg des Ausländeranteils um einen Prozentpunkt das gesamtwirtschaftliche Lohnnivau kurzfristig nur um rund $0,1 \%$ (maximal $0,18 \%$ ) senkt. Langfristig, nach Anpassung des Kapitalstocks, wirkt sich Migration überhaupt nicht auf das Lohnniveau aus.

Allerdings sind die einzelnen Gruppen im Arbeitsmarkt unterschiedlich von der Migration betroffen: Die Löhne der einheimischen Bevölkerung steigen langfristig in allen Studien, die berücksichtigen, dass In- und Ausländer unvollkommene Substitute im Arbeitsmarkt sind. Auf kurze Frist ergeben sich in einem Teil der Studien leichte Gewinne, in einem anderen Teil der Studien leichte Verluste für die Inländer. Umgekehrt sinken die Löhne der bereits im Lande lebenden Ausländer je nach Studie im Durchschnitt erheblich zwischen 0,3 und 3,4 \%. Die Verluste der ausländischen Bevölkerung sind auf zwei Umstände zurückzuführen: Zum einen haben die Zuwanderer häufig einen ähnlichen Bildungshintergrund und eine ähnliche Berufserfahrungen wie die bereits im Lande lebenden Ausländer. Zum anderen sind In- und Ausländer imperfekte Substitute im Arbeitsmarkt. Beides führt dazu, dass die Neuzuwanderer eher mit bereits im Lande lebenden Ausländern als mit Einheimischen im Arbeitsmarkt konkurrieren. 


\section{Übersicht 2: Lohn- und Beschäftigungseffekte der Zuwanderung: Ergebnisse von Regressionsanalysen und strukturellen Modellen auf nationaler Ebene}

\begin{tabular}{|c|c|c|c|c|c|c|c|}
\hline \multirow[t]{2}{*}{ Studie } & \multirow[t]{2}{*}{ Methode } & \multicolumn{3}{|c|}{ Lohneffekt ${ }^{1,3)}$} & \multicolumn{3}{|c|}{ Beschäftigungseffekt ${ }^{2,3)}$} \\
\hline & & Alle & Inländ. & Ausl. & Alle & Inländ. & Ausl. \\
\hline \multicolumn{8}{|c|}{ US-amerikanische und internationale Studien } \\
\hline \multirow[t]{2}{*}{ Borjas (2003), USA } & Regressionsanalyse & $-0,40$ & - & - & $-0,37$ & - & - \\
\hline & Produktionsfunktion & $-0,32$ & - & - & - & - & - \\
\hline \multirow{7}{*}{$\begin{array}{l}\text { Aydemir/Borjas (2005), } \\
\text { Kanada, USA, } \\
\text { Mexiko }\end{array}$} & Regressionsanalyse & & & & & & \\
\hline & Kanada & $-0,32$ & - & - & $-0,24$ & - & - \\
\hline & Mexiko & $-0,56$ & - & - & 0,06 & - & - \\
\hline & USA & $-0,36$ & - & - & $-0,35$ & - & - \\
\hline & $\begin{array}{l}\text { Produktionsfunktion } \\
\text { Kanada }\end{array}$ & $\begin{array}{l}-0,31 \text { bis } \\
-0,75(0,00)\end{array}$ & - & - & - & - & - \\
\hline & Mexiko & $\begin{array}{l}-0,22 \text { bis } \\
-0,66(0,00)\end{array}$ & - & - & - & - & - \\
\hline & USA & $\begin{array}{l}-0,20 \text { bis } \\
-0,59(0,00)\end{array}$ & - & - & - & - & - \\
\hline Ottaviano/Peri (2006) & Produktionsfunktion & $\begin{array}{l}-0,10 \\
(0,00)\end{array}$ & $\begin{array}{c}0,11 \\
(0,16)\end{array}$ & $\begin{array}{l}-1,85 \\
(-1,80)\end{array}$ & - & - & - \\
\hline Ottaviano/Peri (2008) & & $\begin{array}{l}-0,10 \\
(0,00)\end{array}$ & $\begin{array}{l}-0,04 \\
(0,06)\end{array}$ & $\begin{array}{l}-0,68 \\
(-0,58)\end{array}$ & - & - & - \\
\hline \multicolumn{8}{|c|}{ Deutsche Studien } \\
\hline Bonin (2005) & Regressionsanalyse & $-0,10$ & - & - & insignifikant & - & - \\
\hline Brücker/Jahn (2010) & Lohnsetzungsmodell & $-0,18(0,00)$ & $\begin{array}{l}-0,08 \\
(0,11)\end{array}$ & $\begin{array}{l}-1,11 \\
(-1,09)\end{array}$ & $\begin{array}{l}-0,31 \\
(-0,08)\end{array}$ & $\begin{array}{l}-0,09 \\
(0,06)\end{array}$ & $\begin{array}{l}-1,97 \\
(-1,16)\end{array}$ \\
\hline D'Amuri et al. (2008) & Produktionsfunktion & $(0,00)$ & $(0,69)$ & $(-3,42)$ & 0,07 bis 0,33 & $\begin{array}{l}0,10 \\
\text { bis } 0,27\end{array}$ & $\begin{array}{l}0,00 \text { bis } \\
-0,20\end{array}$ \\
\hline Felbermayr et al. (2008) & Lohnsetzungsmodell & - & $\begin{array}{l}-0,02 \\
(0,06)\end{array}$ & $\begin{array}{l}-0,32 \\
(-0,58)\end{array}$ & - & $\begin{array}{l}-0,17 \\
(0,10)\end{array}$ & $\begin{array}{l}-1,88 \\
(-0,34)\end{array}$ \\
\hline
\end{tabular}

1) Veränderung in \% bei einer Erhöhung des Ausländeranteils um einen Prozentpunkt. 2) Veränderung in Prozentpunkten bei einer Erhöhung des Ausländeranteils um einen Prozentpunkt. 3) Langfristige Effekte, d.h. unter Berücksichtigung einer vollkommenen Anpassung des Kapitalstocks, werden in Klammern angegeben. Quelle: Eigene Zusammenstellung und Umrechnung auf Grundlage der genannten Studien.

WSI MitTELUNGEN

\subsection{EIN NEUER ANSATZ ZUR ANALYSE VON BESCHÄFTIGUNGS- EFFEKTEN}

Insbesondere im Kontext Deutschlands und anderer europäischer Länder mit hoher struktureller Arbeitslosigkeit ist eine systematische Berücksichtigung der Beschäftigungswirkungen der Migration notwendig. Zwar berücksichtigen viele Studien die Wirkungen der Migration auf Arbeitslosigkeit oder Beschäftigung, aber in der Regel werden die Effekte getrennt in einfachen Regressionen geschätzt. Das gilt auch für einen Teil der neueren Studien (Aydemir/Borjas 2005; Borjas 2003; D’Amuri et al. 2008) - sofern sie die Beschäftigungseffekte überhaupt berücksichtigen. Tatsächlich werden die Lohn- und Beschäftigungswirkungen der Migration jedoch simultan bestimmt. Studien, die die Lohneffekte der Migration schätzen, als seien Arbeitsmärkte vollkommen, tendieren deshalb dazu, die Wirkungen von Lohnrigiditäten $\mathrm{zu}$ vernachlässigen und die Lohneffekte zu überschätzen.

Eine neue empirische Strategie zur Analyse der Lohn- und Beschäftigungseffekte der Migration haben Brücker/Jahn (2010) auf Grundlage des oben beschrieben Lohnsetzungsmodells entwickelt. Ein ähn- licher Ansatz wird auch von Felbermayr et al. (2008) verfolgt. Der Lohnsetzungsansatz beruht auf der Annahme, dass sich Gewerkschaften und Arbeitgeber zunächst unter Berücksichtigung von Arbeitslosigkeit auf einen Lohn verständigen. Ist der Lohn erst einmal fixiert, passen die Unternehmen ihre Beschäftigung so an, dass die Profite der Firmen maximiert werden. Empirisch werden zunächst die Elastizitäten der Lohnsetzungskurve, also die Korrelation zwischen Lohn und Arbeitslosigkeit, geschätzt, danach die Arbeitsnachfragefunktionen der Unternehmen. Bei beiden wird zwischen verschiedenen Typen von Arbeit unterschieden. Auf Grundlage der geschätzten Parameter kann das Modell dann simultan für die Beschäftigungseffekte und die Löhne gelöst werden. Ähnlich wie in dem Ansatz von Ottaviano/Peri (2006) wurde die Anpassung des Kapitalstocks an die Ausweitung des Arbeitsangebots und der Umstand, dass Ausländer und Inländer imperfekte Substitute im Arbeitsmarkt sein können, berücksichtigt.

Die Studie kommt zu dem Ergebnis, dass die Zuwanderung von einem Prozent das aggregierte Lohnniveau der Bevölkerung kurzfristig um 0,18 \% senkt, aber langfristig sich keine Veränderung ergibt.
Die Arbeitslosigkeit steigt kurzfristig um 0,31 Prozentpunkte, langfristig aber nur um 0,09 Prozentpunkte. Diese Schätzungen beruhen auf der Annahme, dass die Qualifikationsstruktur der Zuwanderer der durchschnittlichen Qualifikationsstruktur der Ausländer in Deutschland während der Periode 1980 bis 2004 entspricht. Unter dieser Annahme würden Gruppen nach Deutschland wandern, die einem erhöhten Arbeitslosigkeitsrisiko ausgesetzt sind, sodass auch die gesamtwirtschaftliche Arbeitslosenrate langfristig leicht ansteigt.

Wichtiger als die durchschnittlichen Wirkungen sind die Effekte für die einzelnen Gruppen im Arbeitsmarkt. Während die einheimischen Arbeitnehmer durch steigende Löhne und geringe Arbeitslosigkeit von der Zuwanderung profitieren, ergeben sich erhebliche Verluste für die bereits im Lande lebenden ausländischen Arbeitnehmer: Ihre Löhne würden bei einer Zuwanderung von einem Prozent der Erwerbspersonen in Deutschland um $1,1 \%$ fallen und ihre Arbeitslosenrate würde um 1,16 Prozentpunkte steigen. Damit kann rund ein Drittel des Anstiegs der Arbeitslosigkeit unter der ausländischen Bevölkerung seit Ende der 1980er Jahre durch Zuwanderung erklärt werden. 
Das Bild würde sich jedoch mit der Qualifikations- und Altersstruktur der Zuwanderung verändern: Je besser die Qualifikation der Zuwanderer und je jünger das Alter der zugewanderten Personen, desto günstiger sind die Arbeitsmarkteffekte. Je nach den Annahmen, die über die Struktur des Zuwanderungsschocks getroffen werden, kann es auch zu einem Rückgang der gesamtwirtschaftlichen Arbeitslosenrate kommen. Da die Qualifikation der Zuwanderer in Deutschland steigt, dürften die tatsächlichen Arbeitsmarktwirkungen der Migration günstiger als in Übersicht 2 skizziert ausfallen.

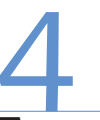

\section{Zusammenfassung und Schlussfolgerungen}

Weite Teile der Bevölkerung erwarten, dass die Zuwanderung von ausländischen Arbeitnehmern zu fallenden Löhnen und steigenden Arbeitslosigkeitsrisiken für die einheimische Bevölkerung führt. Diese Befürchtungen haben einen erheblichen Einfluss auf die Zuwanderungspolitik in Deutschland wie in anderen wichtigen Zielländern der Migration. Eine breite Literatur hat diese Befürchtungen aus theoretischer und empirischer Perspektive untersucht. Aus theoretischer Perspektive zeigt sich, dass eine Ausweitung des Arbeitsangebots durch Zuwanderung nicht unbedingt $\mathrm{zu}$ fallenden Löhnen und - bei unvollkommenen Arbeitsmärkten - zu steigender Arbeitslosigkeit führen muss. Die Anpassung der Kapital- und Produktmärkte kann vielmehr bewirken, dass die Arbeitsmarktwirkungen der Migration neutral sind.

Auf Grundlage dieser theoretischen Hypothesen hat sich in den 1980er und 90er Jahren eine umfangreiche empirische Literatur entwickelt, die zu dem Ergebnis kommt, dass Migration sich entweder neutral auf den Arbeitsmarkt auswirkt oder nur in sehr geringem Umfang zu sinkenden Löhnen und steigender Arbeitslosigkeit führt. Diese Befunde wurden jedoch aufgrund methodischer Probleme bei der Messung der Migrationseffekte vielfach kritisiert. Jüngere Ansätze nutzen nunmehr die Varianz des Ausländeranteils über Qualifikations- und Berufserfahrungsgruppen anstelle der regionalen Varianz des Ausländeranteils, um die Arbeitsmarktwirkungen der Migration zu identifizieren. Diese Strategie hat den Vorteil, dass Endogenitätsprobleme umgangen werden können, die sich daraus ergeben, dass sich Ausländer bevorzugt in Regionen mit hohen Löhnen und niedrigen Arbeitslosigkeitsrisiken niederlassen. Auch wenn einige US-Studien zunächst deutlich höhere Schätzergebnisse ermittelt haben, so bestätigt diese Literatur weitgehend die Ergebnisse der alten Schätzansätze: Die Erhöhung des Ausländeranteils um einen Prozentpunkt - das entspräche in Deutschland einer Nettozuwanderung von rund 820.000 Personen - würde den meisten Studien zufolge das gesamtwirtschaftliche Lohnniveau kurzfristig nur um 0,1 bis $0,2 \%$ senken und die Arbeitslosenrate um 0,1 bis 0,3 Prozentpunkte erhöhen. Langfristig, nach einer Anpassung des Kapitalstocks, ergäben sich keinerlei Auswirkungen auf das gesamtwirtschaftliche Lohnniveau. Die Arbeitslosenrate würde um maximal 0,1 Prozentpunkte steigen - sofern Gruppen mit einem überdurchschnittlichen Arbeitslosigkeitsrisiko zuwandern.

Das wirtschaftspolitisch bedeutsamste Ergebnis dieser Studien ist, dass sich die Migration sehr unterschiedlich auf die einzelnen Gruppen im Arbeitsmarkt auswirkt. Während die einheimische Bevölkerung den meisten Studien zufolge in fast allen Qualifikationsgruppen gewinnt, verlieren die bereits im Lande lebenden Ausländer erheblich. Dies ist zum einen darauf zurückzuführen, dass diese Gruppen häufig ein ähnliches Qualifikationsniveau wie die Neuzuwanderer aufweisen; zum anderen wirkt sich hier aus, dass In- und Ausländer auch bei gleicher Qualifikation und Berufserfahrung nur unvollkommen im Arbeitsmarkt konkurrieren. Insofern hat Migration weniger einen Einfluss auf die Verteilung von Einkommen zwischen Kapital und Arbeit, sondern mehr einen Einfluss auf die Verteilung von Löhnen und Beschäftigungschancen zwischen den einzelnen Arbeitnehmergruppen.

Aus den Befunden der empirischen Literatur ergeben sich auch einige wichtige Schlussfolgerungen für die Einwanderungs- und Integrationspolitik. Die Arbeitsmarktwirkungen der Migration fallen umso positiver aus, je höher das Qualifikationsniveau der Zuwanderer ist. Die seit dem Ölpreisschock von 1973 verfolgte Politik, den Arbeitsmarkt in Deutschland gegen Zuwanderung zu schützen, hatte den unintendierten Nebeneffekt, dass sich durch Familiennachzug und andere Kanäle die Qualifikationsstruktur der Zuwanderer in Deutschland verschlechtert hat. Damit dürften sich die Arbeitsmarktwirkungen der Migration verschlechtert haben. Demgegenüber könnten durch eine Einwanderungspolitik, die zwar mehr Zuwanderung zulässt, aber die Zuwanderung nach Humankapitalkriterien steuert, auch günstigere Wirkungen für den Arbeitsmarkt erzielt werden. Auch der Befund, dass Aus- und Inländer bei gleicher Qualifikation und Berufserfahrung nur unvollkommen im Arbeitsmarkt konkurrieren, hat politische Implikationen. Durch die unvollkommene Konkurrenz werden die besonders verwundbaren Gruppen im Arbeitsmarkt besonders durch Zuwanderung getroffen. Eine Integrationspolitik, die etwa durch die Entwicklung von Sprachkompetenz oder die Anerkennung von beruflichen Bildungsabschlüssen die Integration der Zuwanderer in den Arbeitsmarkt fördert, würde auch zu einem Rückgang der Arbeitslosenraten von Ausländern und in der Gesamtwirtschaft beitragen. 
Aydemir, A./Borjas, G.J. (2005): Cross-Country variation in the Impact of International Migration: Canada, Mexico and the United States, in: Journal of European Economic Association 4, S. 663-708

Bauer, T. (1998): Arbeitsmarkteffekte der Migration und Einwanderungspolitik. Eine Analyse für die Bundesrepublik Deutschland, Heidelberg Bentolila, S./Dolado, J.J./Jimeno, J.F. (2007): Does Immigration Affect the Philipps Curve? Some Evidence for Spain, Kiel Working Papers 1333, Kiel Institute for the World Economy (IfW), Kiel

Binyamini, A./Razin, A. (2008): Inflation-Output Tradeoff as Equilibrium Outcome of Globalization, NBER Working Paper 14379, National Bureau of Economic Research, Cambridge/USA

Boeri, T./Brücker, H. (2005): Why are Europeans so tough on migrants?, in: Economic Policy 44, S. 631-703

Bonin, H. (2005): Wage and Employment Effects of Immigration to Germany: Evidence from a Skill Group Approach, IZA Discussion Paper 1875, Bonn

Borjas, G.J. (2003): The Labour Demand Curve is Downward-Sloping: Reexamining the Impact of Immigration on the Labour Market, in: Quarterly Journal of Economics 4, S. 1335-1374

Borjas, G.J./Freeman, R./Katz, L. (1997): "How much do immigration and trade affect labor market outcomes?", in: Brookings Papers on Economic Activity 1, S. 1-90

Borjas, G.J./Grogger, J./Hanson, G.H. (2008): Imperfect Substitution between Immigrants and Natives: A Reappraisal, NBER Working Paper 13887, National Bureau of Economic Research, Cambridge/USA Brücker, H. (2009): Arbeitsmarktwirkungen der Migration, in: Aus Politik und Zeitgeschichte (APuZ) 44, S. 6-12

Brücker, H./Jahn E. (2010): Migration and Wage Setting: Reassessing the Labor Market Effects of Migration, Scandinavian Journal of Economics, im Erscheinen

Card, D. (1990): The impact of the Mariel boatlift on the Miami labor market, in: Industrial and Labor Relations Review 3, S. 245-257

Card, D. (2001): Immigrant Inflows, Native Outflows, and the Local Labor Market Impacts of Higher Immigration, in: Journal of Labor Economics 1, S. 22-64

Card, D. (2005): Is the New Immigration Really so Bad?, in: Economic Journal 507, S. F300-F323

Card, D./Di Nardo, J. (2000): Do immigrant inflows lead to native outflows?, in: American Economic Review, Papers and Proceedings 4, S. 361-367

Carrington, W./DeLima, P. (1996): The impact of the 1970s repatriates from Africa on the Portuguese labour market, in: Industrial and Labor Relations Review 4, S. 330-346

D'Amuri, F.,/Ottaviano, G./Peri, G. (2008): The Labor Market Impact of Immigration in Western Germany in the 1990's, NBER Working Paper 13851, National Bureau of Economic Research, Cambridge/USA DeNew, J./Zimmermann, K.F. (1994): Native Wage Impacts of Foreign Labor: A Random Effects Panel Analysis, in: Journal of Population Economics 7, S. 177-192

Dustmann, C./Fabbri, F./Preston, I. (2005): The Impact of Immigration on the UK Labour Market, in: Economic Journal 507, S. F324-F341 Felbermayr, G./Geis, W./Kohler, W.(2008): Absorbing German Immigration: Wages and Employment, CESifo Working Paper

Friedberg, R./Hunt, J. (1995): The Impact of Immigrants on Host Country Wages, Employment and Growth, in: Journal of Economic Perspectives 2, S. 23-44
Glitz, A. (2006): The Labor Market Impact of Immigration: QuasiExperimental Evidence, CreAM Discussion Paper 12, Centre for research and Analysis of Migration, London

Haisken-DeNew, J./Zimmerman K.F. (1995): Wage and Mobility Effects of Trade and Migration, CEPR Discussion Paper 1318, Centre for Economic Policy Research, London

Hamermesh, D. (1993): Labor Demand, Princton

Hunt, J. (1992): The impact of the 1962 repatriates from Algeria on the French labor market, in: Industrial and Labor Relations Review 2,

S. 556-572

Kaldor, N. (1961): Capital accumulation and economic growth, in: Lutz, F./Hague, D. (Hrsg.): The Theory of Capital, St. Martins/New York Layard, R./Nickell, S.J./Jackman, R. (2005): Unemployment: Macroeconomic Performance and the Labour Market, Oxford Levine, P. (1999): The welfare economics of migration control, in: Journal of Population Economics 1, S. 23-43

Longhi, S./Nijkamp, P./Poot, J. (2005): A Meta-Analytic Assessment of the Effects of Immigration on Wages, in: Journal of Economic Surveys 3, S. 451-77

Longhi, S./Nijkamp, P./Poot, J. (2006): The Impact of Immigration on the Empoyment of Natives in Regional Labour Markets: A Meta-Analysis, IZA Discussion Paper 2044, Bonn

Longhi, S./Nijkamp, P./Poot, J. (2008): Meta-Analysis of Empirical Evidence on the Labor Market Impacts of Immigration, IZA Discussion Paper 3418, Bonn

Manacorda, M./Manning, A./Wadsworth, J. (2006): The Impact of Immigration on the Structure of Male Wages: Theory and Evidence from Britain, IZA Discussion Paper 2352, Bonn

Mühleisen, M./Zimmermann, K.F. (1994): A panel analysis of job changes and unemployment, in: European Economic Review 7, S. 793-801 Organisation for Economic Co-Operation and Development (OECD) (2010): Main Economic Indicators, Paris

Ottaviano, G./Peri, G. (2006): Rethinking the Effects of Immigration on Wages, NBER Working Paper 12497, Cambridge/USA

Ottaviano, G./Peri, G. (2008): Immigration and National Wages: Clarifying the Theory and the Empirics, NBER Working Paper Nr. 14188, National Bureau of Economic Research, Cambridge/USA

Pischke, J./Velling, J. (1997): Employment effects of immigration to Germany: an analysis based on local labor markets, in: Review of Economics and Statistics 3, S. 594-604

Statistisches Bundesamt (2010), Mikrozensus 2009, Wiesbaden Weltbank (2009): World Development Indicators, CD-Rom, Washington DC.

Winkelmann, R./Zimmermann, K.F. (1993): Ageing, Migration and Labor Mobility, in: Johnson, P./Zimmermann, K.F. (Hrsg.): Labor Markets in an Ageing Europe, Cambridge/UK

Winter-Ebmer, R./Zimmermann, K.F. (1998): East-West Trade and Migration: The Austro-German Case, IZA Discussionpaper 2, Bonn Winter-Ebmer, R./Zweimüller, J. (1996): Immigration and the Earnings of Young Native Workers, Oxford Economic Papers 48, S. 473-491 Wong, K.-Y. (1995): International Trade in Goods and Factor Mobility, Cambridge/USA

Wooldrige, J.M. (2007): Introductory Econometrics: A Modern Approach, New York 